\title{
Sintomas respiratórios em pacientes atendidos em uma unidade básica de saúde de Goiânia-GO
}

\author{
Respiratory symptoms in patients consulting at a primary health care unit of Goiania-GO \\ Sintomas respiratorios en los pacientes tratados en una unidad de atención primaria de Goiânia-GO
}

José Laerte Rodrigues Silva Júnior. Universidade Federal de Goiás (UFG). Goiânia, GO, Brasil. joselaertejr@gmail.com (Autor correspondente)

Thiago Fintelman Padilha. Universidade Federal de Goiás (UFG). Goiânia, GO, Brasil. thiagofintelman@hotmail.com

Jordana Eduardo Rezende. Universidade Federal de Goiás (UFG). Goiânia, GO, Brasil. jojo_rzd@hotmail.com

Eliane Consuelo Alves Rabelo. Universidade Federal de Goiás (UFG). Goiânia, GO, Brasil. elianeconsuelo@gmail.com

Anna Carolina Galvão Ferreira. Universidade Federal de Goiás (UFG). Goiânia, GO, Brasil. annacarolgalvao@gmail.com

Marcelo Fouad Rabahi. Universidade Federal de Goiás (UFG). Goiânia, GO, Brasil. mfrabahi@gmail.com

\section{Resumo}

Objetivo: verificar a prevalência de sintomas respiratórios em indivíduos que procuram uma unidade básica de saúde, descrevendo as distribuições desses sintomas e dos fatores de risco relacionados com doenças respiratórias. Métodos: estudo transversal em uma unidade básica de saúde de Goiânia-GO. Durante um ano, 44 observações, escolhidas de forma aleatória, foram realizadas, onze em cada estação do ano. Para análise dos dados, foram utilizados teste do qui-quadrado, ANOVA, correlação e regressão robusta univariada. Resultados: dentre os 3.354 indivíduos avaliados, 13,7\% (458/3.354) possuíam sintomas respiratórios. A tosse foi o sintoma respiratório mais prevalente, ocorrendo em 91\% (417/458) dos casos. Em relação aos que procuraram a unidade de saúde, 4,8\% (161/3354) apresentavam tosse por duas semanas ou mais. A proporção dos diferentes sintomas respiratórios (tosse, dispneia e chiado) foi semelhante entre as estações, e as prevalências de sintomas respiratórios encontradas nas quatros estações (inverno, outono, verão e primavera) foram, respectivamente, 20\%, 14\%, 11,9\% e 7,4\%. A média do tempo de tosse para idosos foi estatisticamente maior que nos demais grupos $(p=0,004)$. Os tabagistas, ex-tabagistas, portadores de baixo peso, pessoas que referiam pneumonia prévia, asma ou DPOC apresentaram médias de tempo de tosse maiores, mas essas diferenças não foram estatisticamente significativas. 0 modelo de regressão mostrou aumento do tempo de tosse com aumento da idade $\left(r^{2}=0,08 ; p=0,0001\right)$. Conclusão: as doenças respiratórias perfazem uma importante parcela dos atendimentos da unidade básica de saúde. A prevalência de sintomas respiratórios é maior no inverno e 0 tempo médio de tosse aumenta com a idade.

\section{Abstract}

Objective: to determine the prevalence of respiratory symptoms in subjects attending a primary healthcare unit, describing the distributions of these symptoms and of risk factors for respiratory disease. Methods: a cross-sectional study was conducted on subjects attending an outpatient primary health care unit in Goiania-GO. During one year, forty-four random observations categorized by season were made. Chi-square test, analysis of variance, correlation and univariate robust regression were used to perform the statistical analyses. Results: among the 3,354 subjects enrolled, $13.7 \%$ $(458 / 3,354)$ had respiratory symptoms. Cough was the most prevalent symptom, occurring in $91 \%$ (417/458) of the cases. Among all subjects, $4.8 \%$ $(161 / 3,354)$ had cough for more than two weeks. The proportion of respiratory symptoms (cough, dyspnea and wheezing) did not differ significantly across seasons and the prevalence of respiratory symptoms in winter, autumn, summer and spring were, respectively, 20\%, $14 \%, 11.9 \%$ and $7.4 \%$. The average duration of cough in the elderly was significantly longer than in the other age groups $(p=0,004)$. Smokers, former smokers, low weight subjects and subjects reporting previous pneumonia, asthma or COPD also showed longer average duration of cough, but these differences were not statistically significant. The regression model showed that the duration of cough increased with age $\left(r^{2}=0,08 ; p=0,0001\right)$. Conclusion: subjects with respiratory diseases account for a significant proportion of the demand for healthcare. The prevalence of respiratory symptoms is higher during the winter and the average duration of cough increases with age.

Como citar: Silva Júnior JLR, Padilha TF, Rezende JE, Rabelo ECA, Ferreira ACG, Rabahi MF.

\section{Palavras-chave:}

Sinais e Sintomas Respiratórios Transtornos Respiratórios

Centros de Saúde Fatores de Risco 


\section{Resumen}

Objetivo: investigar la prevalencia de personas con síntomas respiratorios que buscan una unidad básica de salud, describiendo sus distribuciones de síntomas y factores correlacionados con las enfermedades respiratorias. Métodos: estudio transversal en una unidad básica de salud en Goiânia-GO. Por un año, se realizaron cuarenta y cuatro observaciones elegidas al azar, once de cada temporada. Para el análisis de los datos se utilizó chi-cuadrado, ANOVA, correlación y regresión robusta univariada. Resultados: de los 3.354 sujetos evaluados, 13,7\% (458/3,354) tenían síntomas respiratorios. La tos fue el síntoma respiratorio más frecuente, presente en 91\% (417/458). Considerando quien buscó la unidad de salud, 4,8\% (161/3354) tuvieran tos durante dos semanas o más. La proporción de los síntomas respiratorios (tos, disnea y sibilancias) no difirió entre temporadas. La prevalencia de síntomas respiratorios que se encuentran en las cuatro estaciones (invierno, otoño, verano y primavera) fueron, respectivamente, 20\%, 14\%, 11,9\% y 7,4\%. La duración media de la tos en ancianos fue estadísticamente mayor que los otros grupos $(p=0,004)$. Los fumadores, ex-fumadores, con bajo peso, que informaron neumonía previa, asma o EPOC tenían duración media de la tos mayor, pero estas diferencias no fueron estadísticamente significativas. El modelo de regresión mostró una mayor duración de la tos con la edad $(r 2=0,08, p=0,0001)$. Conclusión: las personas con enfermedades respiratorias representan una parte importante del cuidado de la unidad básica de salud. La prevalencia de síntomas respiratorios es mayor en invierno y la media de la tos aumenta con la edad.
Palabras clave:

Signos y Síntomas Respiratorios Transtornos Respiratorios Centros de Salud Factores de Riesgo

\section{Introdução}

As doenças respiratórias, comuns em todas as faixas etárias nos países desenvolvidos e em desenvolvimento, têm sido consideradas importantes causas de morbimortalidade, e, nas últimas décadas, sua incidência tem aumentado gradativamente. ${ }^{1}$ As mais frequentes nas unidades básicas de saúde e que resultam em significativas morbidade e mortalidade são as infecçóes respiratórias agudas, a tuberculose, a asma, a doença pulmonar obstrutiva crônica (DPOC) e o câncer de pulmão. A tuberculose e a pneumonia são importantes causas de morbidade e mortalidade em adultos jovens em países de baixa e média renda. Já em países desenvolvidos, a pneumonia e o câncer de pulmão apresentam uma incidência significativa em indivíduos com mais de 50 anos. O ônus da asma tem aumentado em todo o mundo, e a DPOC é uma importante causa de invalidez e morte em toda parte. ${ }^{2}$

Devido à importância das doenças respiratórias em termos de saúde pública, a Organização Mundial de Saúde (OMS) sugeriu em 2005 uma estratégia voltada para países em desenvolvimento que permitisse a abordagem e o manejo integrado dos pacientes com cinco anos de idade ou mais que se apresentassem em unidades de atenção primária com sintomas respiratórios. ${ }^{2}$ Essa abordagem sistematizada é chamada de estratégia PAL (Practical Approach to Lung Health) e consiste em uma abordagem sindrômica por meio da utilização de algoritmos. Assim, com base tanto em sinais e sintomas respiratórios, quanto na sua duração e em seus fatores de risco, o algoritmo de atendimento orienta o profissional de saúde nas açóes diagnósticas e terapêuticas a serem tomadas no nível de atendimento primário. ${ }^{3}$

Um estudo realizado em Goiânia verificou que a prevalência de asma em adolescentes de 13 e 14 anos era de 10,6\%, um achado similar ao encontrado em outras capitais brasileiras. ${ }^{4}$ Em relaçáo à tuberculose, a incidência no estado de Goiás em 2009 foi de 14,6/100.000 habitantes, uma das menores taxas em relação ao restante do país. ${ }^{5}$ Ainda não há publicações no que se refere à prevalência de DPOC, mas a prevalência de tabagismo na cidade $(16 \%)^{6}$ é próxima à nacional $(17,2 \%) .^{7}$ Para adaptação e desenvolvimento da estratégia PAL, é necessário identificar o padrão demográfico e a situaçáo epidemiológica da população a ser assistida. Esse conhecimento permite inferir as morbidades mais prevalentes na comunidade, traçar objetivos e, após a implantaçấo, verificar as metas atingidas. ${ }^{3}$ Assim, o objetivo deste trabalho foi identificar a prevalência de pacientes com sintomas respiratórios que procuram uma unidade básica de saúde, descrevendo suas distribuições de sintomas e de fatores de risco para doenças respiratórias.

\section{Métodos}

O estudo foi realizado em uma unidade básica de saúde de Goiânia, Novo Horizonte, responsável pelo atendimento de aproximadamente 40 bairros da cidade, perfazendo um total de 170.000 habitantes. Como era esperada variação sazonal na prevalência de sintomas respiratórios, optou-se pela realização de cortes transversais de igual número nas quatro estaçôes do ano. Por falta de dados nacionais com relação a sintomáticos respiratórios na população maior de cinco anos, o cálculo do tamanho da amostra foi realizado com base na prevalência média observada (18\%) em um estudo da OMS realizado em 9 países em desenvolvimento. ${ }^{8}$ Com nível de confiança de $95 \%$ e com precisão relativa de 7\%, uma amostra de 3.136 indivíduos seria 
necessária. ${ }^{9}$ Como a Unidade de Saúde esperava um atendimento anual de 28.000 indivíduos, com quarenta observaçóes (10 em cada estaçáo do ano) seria possível atingir a amostra desejada. Para garantir a quantidade necessária para o estudo, optou-se por 11 observaçóes em cada estaçáo do ano, realizadas no período de 8 de janeiro a 17 de dezembro de 2009. Os dias de corte transversal foram escolhidos de forma aleatória por meio do uso de um módulo do Microsoft Office Excel de geração de números aleatórios únicos (RandLotto) associado à função do programa Stata versão 11.0 (StataCorp, Texas, USA) para conversão dos números em datas. As observaçóes transversais ocorreram tanto nos dias de semana quanto nos finais de semana, e cada observação durou 12 horas (das 7:00 às 19:00). Durante o período de observação, era realizada uma entrevista de triagem, na qual todos os indivíduos com mais de 5 anos de idade que compareceram à unidade de saúde por qualquer razão eram entrevistados por meio de 6 perguntas fechadas: 1) Nome; 2) Sexo; 3) Idade; 4) Presença de tosse; 5) Presença de falta de ar; 6) Presença de chiado. Os indivíduos que eram considerados elegíveis por apresentar qualquer sintoma respiratório eram convidados a participar. Aqueles indivíduos que tiveram seu Termo de Consentimento Livre e Esclarecido (TCLE) assinado foram incluídos, submetidos a um segundo instrumento de coleta de dados e acompanhados até a alta da unidade de saúde. O segundo instrumento de coleta de dados consistia de um formulário com campos para anotar os dados antropométricos após a aferição de peso e altura; a temperatura axilar medida com termômetro de coluna de mercúrio; os dados relacionados ao tabagismo segundo os critérios adotados no Eurobarometer on Tobacco Survey em $2008 ;{ }^{10}$ os dados relacionados a diagnósticos autorreferidos de bronquite crônica, enfisema, asma, rinite/sinusite, pneumonia prévia e tuberculose em tratamento ou no passado; dados sobre febre autorreferida; histórico de procura do posto de saúde previamente, com ou sem os mesmos sintomas; percepção do estado de saúde na alta da unidade de saúde (melhora, piora, inalterado); e diagnóstico final anotado no prontuário médico pelo médico assistente. O Quadro 1 resume as variáveis aferidas no estudo, bem como suas definiçôes. Foram excluídos do estudo os indivíduos que não completaram a coleta de dados ou que não puderam ser acompanhados até a alta da unidade de saúde ou hospitalização pelo médico assistente. Os dados foram coletados por quatro estudantes de medicina do quarto ano treinados especificamente para esse propósito usando instrumento de coleta de dados específico do estudo. Para garantir que todos os indivíduos que compareceram à unidade de saúde fossem entrevistados, a equipe de coleta de dados foi posicionada próxima à entrada principal.

Quadro 1. Variáveis e definições para o estudo.

\begin{tabular}{ll}
\hline \multicolumn{1}{c}{ Variáveis } & \multicolumn{1}{c}{ Definições para o estudo } \\
\hline Indivíduo com sintomas respiratórios & Indivíduo com tosse e/ou falta de ar e/ou chiado \\
Indivíduo sem sintomas respiratórios & Indivíduo sem tosse, sem falta de ar e sem chiado \\
Sintomático respiratório & Indivíduo com tosse por três semanas ou mais \\
Criança & Indivíduo com idade igual ou inferior a 9 anos \\
Adolescente & Indivíduo com idade entre 10 e 19 anos \\
Adulto & Indivíduo com idade entre 20 e 64 anos \\
Idoso & Indivíduo com 65 anos ou mais \\
Tabagista em atividade & Todo indivíduo que fuma, independentemente da frequência e da intensidade \\
Ex-tabagista & Todo indivíduo que não fuma mas que já fumou em alguma época de sua vida \\
Não tabagista & Indivíduo que nunca fumou \\
Fumante passivo & Indivíduo não tabagista que mora com fumante que fuma dentro \\
& do domicílio e/ou indivíduo não tabagista que durante o trabalho \\
& se expõe diariamente à fumaça de cigarro \\
\hline
\end{tabular}

O teste do qui-quadrado foi utilizado para analisar variáveis dicotômicas. A análise de variância (ANOVA) foi usada para a comparação de três ou mais médias, e o teste de Bartlett foi realizado para confirmar o pré-requisito de variâncias estatisticamente iguais para o emprego da ANOVA. A análise de correlação foi empregada para estudar o comportamento conjunto das variáveis quantitativas, e a regressão robusta univariada foi selecionada para explorar a relação entre as variáveis dependentes e variáveis independentes específicas, por contornar as limitaçóes do método dos mínimos quadrados em relação a heteroscedasticidade e outliers. Os resultados foram analisados com o programa STATA, versão 11.0 (Stata Corp, College Station, TX, EUA). Para todos os testes, valores de $\mathrm{p}<0,05$ foram considerados estatisticamente significativos. O estudo foi aprovado pelo Comitê de Ética em Pesquisa Humana e Animal do Hospital das Clínicas da Universidade Federal de Goiás sob o número 142/2008. 


\section{Resultados}

A entrevista foi apresentada a 3.695 indivíduos (média de 76 entrevistas por dia nas 44 observaçóes), sendo que 8,1\% (299/3695 indivíduos) recusaram-se a participar do estudo. A Figura 1 demonstra o fluxo dos indivíduos.

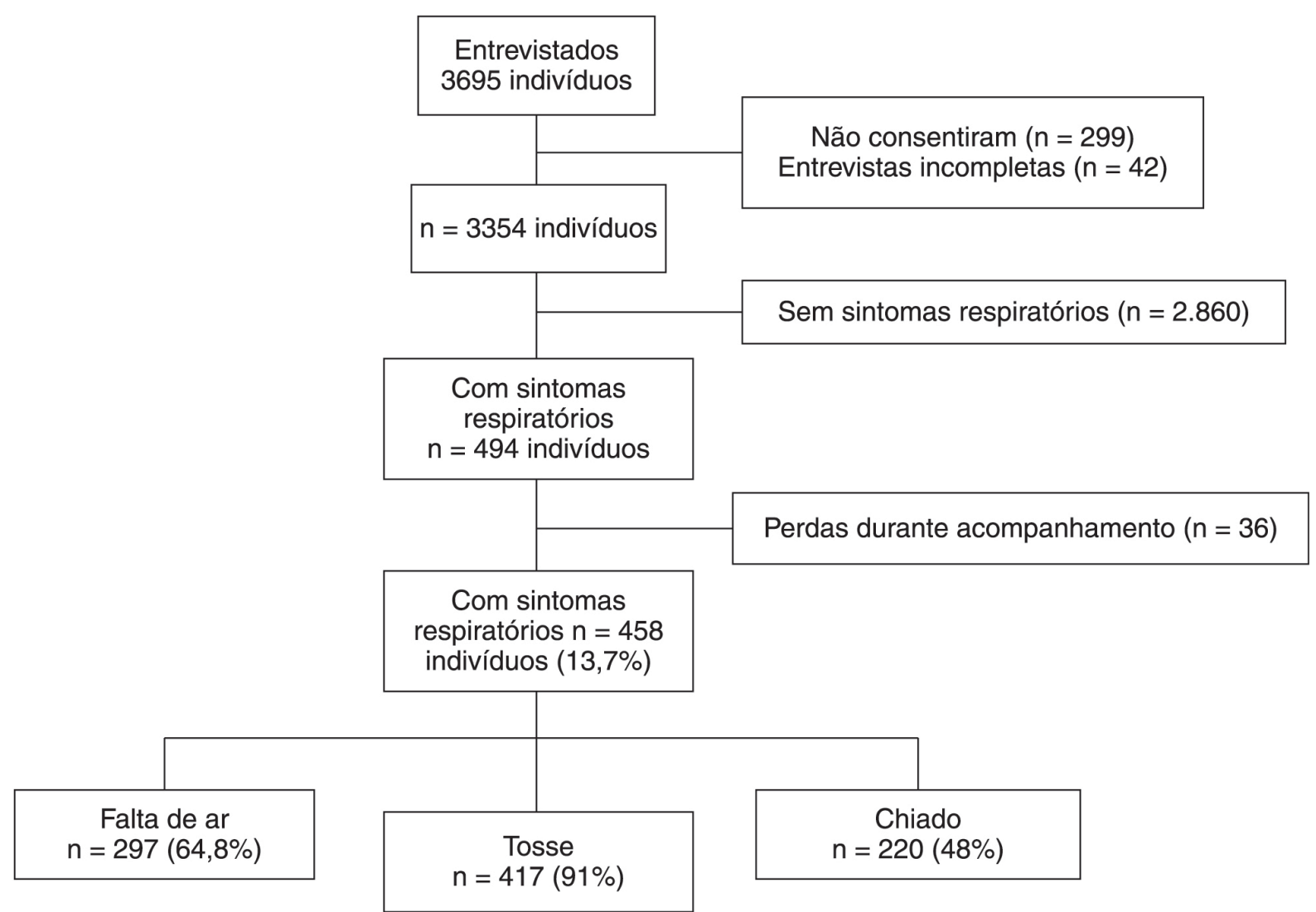

Figura 1. Fluxograma de entrevistas dos indivíduos que procuram a unidade básica de saúde nos dias de corte transversal.

Dentre os 458 indivíduos que procuraram a unidade de saúde com sintomas respiratórios (13,7\%), a tosse foi o sintoma respiratório mais prevalente, presente em 417 indivíduos (91\%), seguido por falta de ar em 297 indivíduos (64,8\%) e chiado em 220 (48\%). Houve predominância de gênero (56,4\% do sexo feminino), e a distribuição por faixa etária foi a seguinte: $\leq 9$ anos, 51 indivíduos (11,1\%); entre 10 e 19 anos, 95 indivíduos (20,7\%); entre 20 e 64 anos, 254 indivíduos (55,5\%); $\geq 65$ anos, 58 indivíduos (12,7\%). A mediana de idade era de 28 anos, a maioria dos indivíduos referia febre $(406 / 88,6 \%)$, $67(14,6 \%)$ dos indivíduos com sintomas respiratórios eram tabagistas em atividade e 139 (30,4\%) apresentavam história de tabagismo no passado ou de tabagismo passivo (Tabela 1).

Em relação a todos os indivíduos avaliados, 4,8\% (161/3354) procuraram a unidade de saúde com duas ou mais semanas de tosse e 2,8\% (94/3354) com três semanas ou mais de tosse. Os indivíduos com 3 semanas ou mais de tosse (94/3354) diferiam dos indivíduos com sintomas respiratórios em geral (364/3354) em relação a: maior idade, maior proporção de tosse produtiva e com sangue, maior proporção de dispneia e chiado, maior proporção de baixo peso, menor proporção de diagnóstico de IVAS (infecção de vias aéreas superiores) e maior proporçáo de diagnóstico de asma e DPOC (Tabela 2).

Dentre os 13,7\% (458/3354) de indivíduos com sintomas respiratórios, 66,6\% (305/458) referiram até uma semana de tosse; $12,9 \%$ (59/458) duas semanas; $10,7 \%$ (49/458) três semanas; e 9,8\% (45/458) quatro semanas ou mais. No entanto, a prevalência de sintomas respiratórios e de indivíduos com tosse por três ou mais semanas na amostra estudada variou nas quatro estaçôes e foi respectivamente de: 20\% (185/926) e 3,5\% (32/926) no inverno; 14\% (126/900) e 2,6\% (23/900) no outono; $11,9 \%$ (90/756) e 2,3\% (17/756) no verão; e 7,4\% (57/772) e 1,9\% (15/772) na primavera. Em relação ao histórico de procura do posto de saúde previamente, 175 indivíduos $(35,4 \%)$ referiam já ter procurado a unidade básica de saúde por sintomas respiratórios. Destes, 134 (76,6\%) procuraram a unidade básica de saúde com os mesmos sintomas. 
Tabela 1. Estatística descritiva do número de entrevistas na unidade básica de saúde em 2009 e características dos indivíduos com sintomas respiratórios.

\begin{tabular}{|c|c|c|c|c|}
\hline \multirow{2}{*}{ Variáveis } & \multirow{2}{*}{ Total (\%) } & \multicolumn{3}{|c|}{ Parâmetros por dia de entrevista } \\
\hline & & Média \pm DP & Mediana (IQR) & Min-Máx \\
\hline Total de indivíduos & $3354(100,0)$ & $76,2 \pm 47,7$ & $60(70)$ & $19-209$ \\
\hline Indivíduos sem sintomas respiratórios & $2860(85,4)$ & $65 \pm 41,8$ & $52(54)$ & $15-185$ \\
\hline Indivíduos com sintomas respiratórios & $458(13,7)$ & $11,2 \pm 10,3$ & $7,5(10)$ & $0-45$ \\
\hline Sexo feminino & $259(56,6)$ & - & - & - \\
\hline Idade & - & $33 \pm 20$ & $28(27)$ & $5-92$ \\
\hline Presença de tosse & $417(91,0)$ & - & - & - \\
\hline Tempo de tosse em dias & - & $10,3 \pm 19,7$ & $4,0(8,0)$ & $1-200$ \\
\hline Presença de dispneia & $297(64,8)$ & - & - & - \\
\hline Tempo de dispneia em dias & - & $9,0 \pm 18,7$ & $4,0(6,0)$ & $1-180$ \\
\hline Presença de chiado & $220(48,0)$ & - & - & - \\
\hline Tempo de chiado em dias & - & $7,9 \pm 13,8$ & $3,0(6,0)$ & $1-120$ \\
\hline Presença de febre & $406(88,6)$ & - & - & - \\
\hline Tabagista em atividade & $67(14,6)$ & - & - & - \\
\hline Ex-tabagista & $50(11,0)$ & - & - & - \\
\hline Fumante passivo & $89(19,5)$ & - & - & - \\
\hline
\end{tabular}

DP = desvio padrão; IQR= amplitude interquartil; Min= valor mínimo; Máx= valor máximo.

Tabela 2. Características dos indivíduos com sintomas respiratórios (geral) e sintomáticos respiratórios (tosse $>3$ semanas) atendidos na unidade de saúde em 2009.

\begin{tabular}{|c|c|c|c|}
\hline Variáveis & $\begin{array}{l}\text { Sintomas respiratórios (geral) } \\
\qquad(n=364)\end{array}$ & $\begin{array}{c}\text { Sintomáticos respiratórios (tosse } \geq 3 \\
\text { semanas) }(n=94)\end{array}$ & $p$ \\
\hline Sexo feminino, n (\%) & $206(56,6)$ & $53(56,4)$ & 0,9 (NS) \\
\hline Idade, mediana (IQR) & $26,5(24,0)$ & $39,5(41,0)$ & $0,005^{\star \star}$ \\
\hline Tosse produtiva, n (\%) & $167(46,0)$ & $54(58,1)$ & $0,04^{*}$ \\
\hline Tosse com sangue, $n(\%)$ & $12(3,4)$ & $10(11,4)$ & $0,002^{*}$ \\
\hline Presença de dispneia, n (\%) & $224(61,6)$ & $73(77,7)$ & $0,004^{*}$ \\
\hline Tempo de dispneia, dias, mediana (IQR) & $1(3,0)$ & $7(15,0)$ & $<0,0001^{* *}$ \\
\hline Presença de chiado, n (\%) & $161(44,2)$ & $59(62,8)$ & $0,001^{*}$ \\
\hline Tempo de chiado, dias, mediana (IQR) & $0(2,0)$ & $2(15,0)$ & $<0,0001^{\star *}$ \\
\hline Presença de febre medida, n (\%) & $87(24,0)$ & $21(22,3)$ & 0,16 (NS) \\
\hline Tabagista em atividade, n (\%) & $48(13,2)$ & $19(20,2)$ & 0,08 (NS) \\
\hline Ex-tabagista, $n(\%)$ & $41(11,3)$ & $9(9,6)$ & 0,64 (NS) \\
\hline Fumante passivo, n (\%) & $72(19,8)$ & $17(18,1)$ & 0,71 (NS) \\
\hline Baixo peso, n (\%) & $30(8,2)$ & $16(17,0)$ & 0,01 * \\
\hline Diagnóstico de IVAS & $127(34,9)$ & $16(17,0)$ & $0,001^{*}$ \\
\hline Presença de asma/DPOC, n (\%) & $20(5,5)$ & $16(17,0)$ & $<0,0001^{*}$ \\
\hline Primavera, n (\%) & $50(13,7)$ & $7(7,5)$ & 0,10 (NS) \\
\hline Verão, n (\%) & $75(20,6)$ & $15(15,9)$ & 0,31 (NS) \\
\hline Outono, n (\%) & $96(26,4)$ & $30(31,9)$ & 0,28 (NS) \\
\hline Inverno, n (\%) & $143(39,3)$ & $42(44,7)$ & $0,34(\mathrm{NS})$ \\
\hline
\end{tabular}

$\mathrm{n}=$ número de indivíduos; IQR= amplitude interquartil; * teste de qui-quadrado estatisticamente significativo; ${ }^{*}$ teste de Mann-Whitney estatisticamente significativo; NS= não significativo; IVAS= infecção de vias aéreas superiores.

Dos 458 indivíduos, 53 (11,6\%) referiam ser portadores de asma ou DPOC, 33 (7,2\%) referiam rinite, $22(4,8 \%)$ ter tido pneumonia previamente e $6(1,3 \%)$ tuberculose pulmonar no passado. A maior parte dos indivíduos estudados 267/458 $(58,3 \%)$ apresentava o índice de massa corpoórea normal, sendo que 90 (19,7\%) estavam com sobrepeso, 55 (12\%) eram obesos e $46(10 \%)$ estavam com baixo peso. 
Houve um predomínio de indivíduos com sintomas respiratórios no inverno, mas a proporção de sintomas respiratórios (tosse, dispneia e chiado) não diferiu estatisticamente entre as estações. Uma diferença estatisticamente significativa entre as médias do tempo de tosse em dias foi observada quando as médias foram avaliadas por faixa etária ( $\mathrm{p}=0,004)$. A média do tempo de tosse para idosos foi estatisticamente maior que as demais. Os tabagistas, ex-tabagistas e indivíduos com baixo peso, bem como aqueles que referiam ter apresentado pneumonia prévia e ser portadores de asma ou DPOC apresentaram médias de tempo de tosse maiores, mas essas diferenças não foram estatisticamente significativas (Gráfico 1).
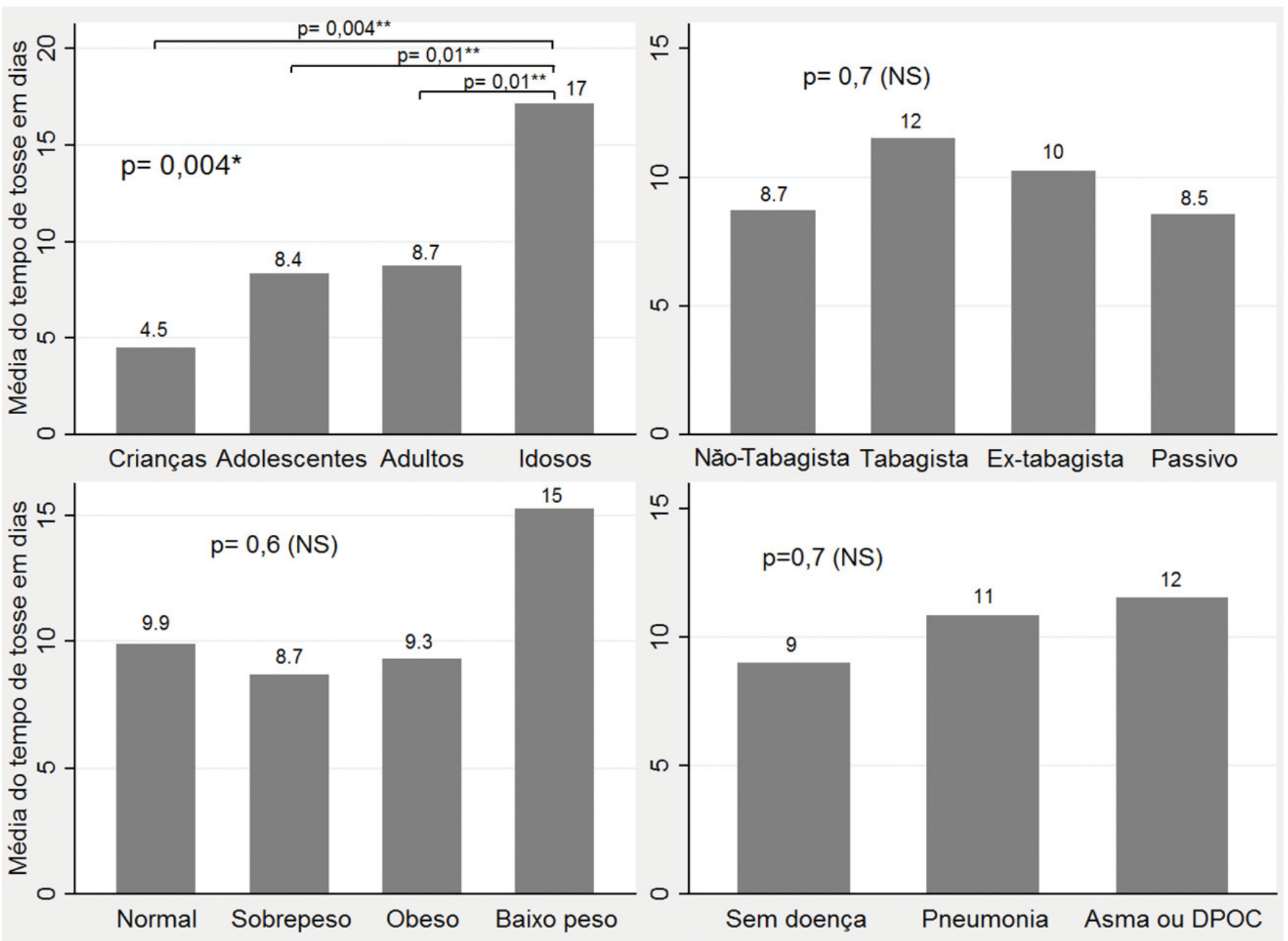

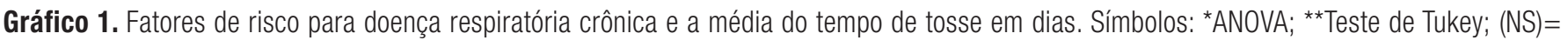
não significativo.

Observou-se uma correlaçáo estatisticamente significativa entre a idade dos indivíduos e o tempo de tosse (coeficiente, 0,27; $\mathrm{p}<0,0001$ ); dispneia (coeficiente, 0,13; $\mathrm{p}<0,05$ ) e respiração ruidosa (coeficiente, 0,16; $\mathrm{p}<0,009$ ). O modelo de regressão mostrou aumento do tempo de tosse com aumento da idade (tempo de tosse $=2,34+0,059$.idade $/ \mathrm{r}^{2}=0,08 /$ $\mathrm{p}<0,0001)$. Essa relação era mais pronunciada em caso de tosse produtiva (Tempo de tosse seca $=1,59+0,06$.idade $/$ $\mathrm{r}^{2}=0,08 / \mathrm{p}=0,0006$, versus, Tempo de tosse produtiva $=2,48+0,41$.idade $\left./ \mathrm{r}^{2}=0,04 / \mathrm{p}=0,001\right)$.

Dos 458 pacientes acompanhados até a alta, $334(72,9 \%)$ receberam alta sem melhora ou com piora do quadro clínico, $108(23,6 \%)$ receberam alta com melhora e $16(3,5 \%)$ foram hospitalizados. O diagnóstico estava anotado no prontuário no momento da alta para 243 indivíduos $(53,1 \%)$. Destes, $143(58,9 \%)$ tiveram o diagnóstico de infecção de via aérea superior, $32(13,2 \%)$ tiveram o diagnóstico de pneumonia, $23(9,5 \%)$ foram diagnosticados como portadores de doença pulmonar obstrutiva crônica, 13 (5,3\%) foram diagnosticados como asmáticos e 32 (13,1\%) tiveram outros diagnósticos. 


\section{Discussão}

Não há publicação nacional avaliando a prevalência de sintomas respiratórios em indivíduos com mais de 05 anos, os quais compóem a população alvo para a estratégia PAL. ${ }^{3}$ A maioria dos estudos realizados no Brasil avaliou indivíduos com 15 anos ou mais. ${ }^{11,12,13}$ Entretanto, a prevalência de sintomas respiratórios (13,7\%) foi semelhante à encontrada nos estudos realizados no país, que variou de 11,3 a $20 \%,{ }^{11,12,13} \mathrm{e}$ em um estudo realizado pela OMS em nove países em desenvolvimento (18\%). ${ }^{8}$ Em Belém do Pará, um estudo que avaliou 1.008 indivíduos em um dia de inverno verificou uma prevalência de tosse de $20 \%$, sendo que $10,3 \%$ destes indivíduos apresentavam tosse por mais de 3 semanas. ${ }^{11}$ Em Vitória, observou-se uma prevalência de sintomas respiratórios de 11,3\% e 4\% de sintomáticos respiratórios na avaliação de 1.101 indivíduos que procuraram unidades básicas de saúde do município no verão e no outono. ${ }^{12}$ Em Curitiba, um estudo que avaliou 2.297 indivíduos com idade entre 2 e 85 anos encontrou uma prevalência de sintomas respiratórios de 14\% e de sintomáticos respiratórios de $2,9 \% \cdot{ }^{13}$ No Distrito Federal, utilizando-se uma metodologia diferente (inquérito domiciliar), um estudo verificou que dentre os 629 indivíduos entrevistados durante o verão, a prevalência de sintomas respiratórios não foi relatada, mas a prevalência de sintomáticos respiratórios variou de 4,8\% a 5,7\% dependendo da área estudada. ${ }^{14}$ No presente estudo, a prevalência de sintomáticos respiratórios foi de 2,8\%, abaixo da prevalência estimada pelo Ministério da Saúde $(5 \%)^{15}$ e da encontrada na maioria dos estudos (4 a 10,3\%). ${ }^{11,12,14}$ Entretanto, a mesma é bem próxima da prevalência encontrada no estudo realizado em Curitiba, cuja amostra era composta por indivíduos a partir de 02 anos, ${ }^{13}$ o que pode explicar a semelhança entre os achados.

Este estudo verificou um aumento dos sintomas respiratórios durante o inverno, com uma prevalência de $23 \%$ contra $12,9 \%$ no outono, $12,4 \%$ no verão e $9,1 \%$ na primavera. No estado de Goiás, o inverno é caracterizado por baixa quantidade de precipitaçáo e baixa umidade relativa do ar. A baixa umidade do ar é considerada um risco para a integridade da via aérea por alterar o equilíbrio do aparelho respiratório, ${ }^{16}$ sendo esse o fator responsável pelo aumento do número de indivíduos com sintomas respiratórios no inverno. ${ }^{17}$

Foi observada a predominância do gênero feminino $(56,4 \%)$ na população que frequenta a unidade de saúde. Em estudos que avaliam serviços de atenção primária, esse é o achado habitual. ${ }^{8,11,12,13}$ Já foi sugerido que as mulheres são mais conscientes em relação à procura de assistência médica quando necessária, além de possuírem piores condiçôes financeiras em relação ao sexo masculino para procurar assistência médica fora do serviço público. ${ }^{18}$

A elevada prevalência de febre observada $(88,6 \%)$ entre os pacientes com sintomas respiratórios no estudo indica uma grande proporção de doenças transmissíveis e está em concordância com o estudo da OMS que verificou que pelo menos $80 \%$ dos indivíduos com sintomas respiratórios avaliados nos países em desenvolvimento apresentam infecção aguda de via aérea. ${ }^{8} \mathrm{O}$ presente estudo também observou uma considerável prevalência de doenças respiratórias crônicas referidas, já que a asma, DPOC e a rinite referidas somadas correspondiam a 23,8\% dos indivíduos avaliados. Como o diagnóstico é referido, provavelmente a taxa real seja maior devido ao subdiagnóstico dessas doenças no Brasil. ${ }^{19,20,21}$ Apesar de não ser possível uma comparaçáo direta entre os dados do estudo e os dados da Pesquisa Nacional por amostra de domicílios em $2008^{7}$ devido à utilização de diferentes definiçóes de tabagismo, a prevalência de tabagismo em atividade encontrada $(14,6 \%)$ estava próxima à verificada pelo IBGE (Instituto Brasileiro de Geografia e Estatística) para a região centro-oeste (16,6\%) e um pouco abaixo da média nacional (17,2\%). ${ }^{7}$ Já a prevalência de tabagismo passivo (19,5\%) estava abaixo da verificada pelo IBGE para região centro-oeste $(22,5 \%)$ e da média nacional $(22,8 \%){ }^{7}$

A Aliança Global Contra Doenças Respiratórias Crônicas da OMS (WHO GARD) reconhece como fatores de risco para prevenção primária o tabagismo em atividade, tabagismo passivo, desnutrição, infecçóes respiratórias de repetição e poluiçáo ambiental. ${ }^{22}$ No entanto, na amostra em estudo, apesar de as médias de tempo de tosse serem maiores para os indivíduos tabagistas, ex-tabagistas, com baixo peso, que referiam ter apresentado pneumonia prévia e ser portadores de asma ou DPOC, essas diferenças não foram estatisticamente significativas. Provavelmente, com uma amostra maior, a diferença numérica encontrada seria estatisticamente significativa.

A análise de regressão evidenciou uma relação entre a idade e o tempo de tosse, o que pode ser explicado pelo aumento da prevalência de doenças respiratórias crônicas com o aumento da idade e pelo agravamento da doença respiratória crônica já presente associado ao envelhecimento. ${ }^{23,24}$ 


\section{Conclusões}

Este estudo confirma que os indivíduos com doenças respiratórias perfazem uma importante parcela dos atendimentos da unidade básica de saúde (aproximadamente $1 \mathrm{em}$ cada 7 indivíduos). A ausência de diagnóstico anotado no prontuário do paciente no final do atendimento pode ser em parte explicada pela inespecificidade dos quadros clínicos atendidos na atenção básica, que muitas vezes não se enquadram nos quadros nosológicos da Classificação Internacional de Doenças (CID). No entanto, a ausência de um registro de morbidade em 46,9\% dos casos é um dado que indica a falta de normatização no atendimento ao paciente com doença respiratória. A implementação da estratégia PAL nessas condiçóes poderá promover padronização do atendimento por meio de capacitação e da utilização de diretrizes para prática clínica, mellhorando a qualidade e a eficiência do atendimento prestado. Dois pré-requisitos para a implantação da estratégia PAL foram alcançados pelo presente estudo: o reconhecimento da situaçáo epidemiológica e a avaliação basal do atendimento prestado aos indivíduos com sintomas respiratórios. Futuros trabalhos serão necessários para avaliar o impacto da implantação dessa estratégia de atendimento na saúde da população.

\section{Agradecimentos}

Agradecemos aos gestores, à toda a equipe de profissionais do Cais Novo Horizonte e à Sociedade Brasileira de Pneumologia e Tisiologia o apoio na realizaçáo deste estudo, ao Prof. Dr. Marcus Barreto Conde pelas sugestóes no preparo desse manuscrito e ao programa Hopkins-Brazil International Clinical Operational and Health Services Research and Training Award (ICOHRTA), financiado pelo Fogarty International Center (Bolsa USNIH \# U2RTW006885 ICOHRTA) que forneceu treinamento ao primeiro autor do manuscrito.

\section{Referências}

1. Aitt-Khaled N, Enarson D, Bousquet J. Chronic respiratory diseases in developing countries: the burden and strategies for prevention and management. Bull World Health Organ. 2001;79(10):971-9.

2. World Health Organization (WHO). Practical Approach to Lung Health (PAL). A primary health care strategy for the integrated management of respiratory conditions in people of five years of age and over. Geneva, Switzerland: WHO; 2005. Disponível em: http://whqlibdoc.who.int/hq/2005/WHO_HTM_TB_2005.351.pdf

3. World Health Organization (WHO). Practical Approach to Lung Health - Manual on initiating PAL implementation. Geneva, Switzerland: WHO; 2008. Disponível em: http://whqlibdoc.who.int/hq/2008/WHO_HTM_TB_2008.410_eng.pdf

4. Costa LDC, Condino Neto A. Prevalência de asma e sintomas relacionados, em adolescentes de Goiânia avaliados pelo questionário ISAAC. Rev Bras Alergia Imunopatol. 2005;(28):309-314.

5. Ministério da Saúde (BR), Secretaria de Vigilância em Saúde. Informe técnico de Tuberculose. Brasil; 2010 Jul [acesso em 2012 Jan 16] Disponível em: http://portalpbh.pbh.gov.br/pbh/ecp/files.do?evento=download\&urlArqPIC=informe_PNCT_TB_julho2010.pdf

6. Carnelosso ML, Barbosa MA, Porto CC, Silva SA, Carvalho MM, Oliveira ALI. Prevalência de fatores de risco para doenças cardiovasculares na região leste de Goiânia (GO). Ciênc Saúde Coletiva [Internet]. 2010 [acesso em 2012 Feb 6];15(1):1073-80. Disponível em: http://dx.doi.org/10.1590/S1413-81232010000700014

7. Instituto Brasileiro de Geografia e Estatística; Instituto Nacional de Câncer (INCA). Pesquisa Nacional por Amostra de Domicílios (PNAD). Tabagismo, 2008. Rio de Janeiro; 2009. Disponível em: http://www1.inca.gov.br/inca/Arquivos/publicacoes/tabagismo.pdf

8. World Health Organization (WHO). Respiratory care in primary care services - A survey in 9 countries. Geneva, Switzerland: WHO; 2004. Disponivel em: http://whqlibdoc.who.int/hq/2004/WHO_HTM_TB_2004.333.pdf

9. Lwanga SK, Lemeshow S. Sample size determination in health studies: a pratical manual. Geneva, Switzerland: WHO; 1991.

10. European Commision. The Gallup Organisation. Survey on Tobacco. Analytical report. Flash Eurobarometer 253. Brussels: European Commision; 2009. Disponível em: http://ec.europa.eu/health/ph_determinants/life_style/Tobacco/keydo_tobacco_en.htm

11. Rodrigues ILA, Cardoso NC. Detecção de sintomáticos respiratórios em serviços de saúde da rede pública de Belém, Pará. Rev Pan-Amaz Saúde. 2010;1(1):67-71. http://dx.doi.org/10.5123/S2176-62232010000100010

12. Moreira CMM, Zandonade E, Lacerda T, Maciel ELN. Sintomáticos respiratórios nas unidades de atenção primária no Município de Vitória, Espírito Santo, Brasil. Cad Saúde Pública. 2010;26(8):1619-26. http://dx.doi.org/10.1590/S0102-311X2010000800015

13. Gabardo BMA, Rossoni AMO, Costa HMLM, Schichta BS, Schlichting GCB, Gomes DC, et al. Prevalência de sintomáticos respiratórios em Unidades de Saúde. In: Anais do III Encontro Nacional de Tuberculose. Brasília: Sociedade Brasileira de Pneumologia e Tisiologia; 2008. p. R21. 
14. Freitas FTM, Yokota RTC, Castro APB, Andrade SSCA, Nascimento GL, Moura NFO, et al. Prevalência de sintomáticos respiratórios em regiões do Distrito Federal, Brasil. Rev Panam Salud Publica. 2011;29(6):451-6. http://dx.doi.org/10.1590/S1020-49892011000600011

15. Ministério da Saúde (BR). Manual de Recomendações para o Controle da Tuberculose no Brasil. Brasília, DF; 2010.186 p. Disponível em: http://www.paho.org/bra/index.php?option=com_docman\&task=doc_details\&gid=1042\&ltemid=423

16. Ahrens CD, Samson PJ. Temperature and Humidity extremes. In: Ahrens CD, Samson PJ, editors. Extreme Weather and Climate. Belmont, CA: Cengage Learning; 2010. p. 86.

17. Silva Júnior JLR, Padilha TF, Rezende JE, Rabelo ECA, Ferreira ACG, Rabahi MF. Efeito da sazonalidade climática na ocorrência de sintomas respiratórios em uma cidade de clima tropical. J Bras Pneumol. 2011;37(6):759-67. http://dx.doi.org/10.1590/S1806-37132011000600009

18. Rangan S, Uplekar M. Gender perspectives of access to health and tuberculosis care. In: Diwan VK, Thorson A, Winkvist A, (editors). Gender and tuberculosis: an international research workshop. Report from the workshop at the Nordic School of Public Health: Göteborg; 1998 May 24-26, p. 107-125.

19. Godoy I, Tanni SE, Coelho LS, Martin RSS, Parenti LC, Andrade LM, et al. Programa de cessação de tabagismo como ferramenta para o diagnóstico precoce de doença pulmonar obstrutiva crônica. J Bras Pneumol. 2007;33(3):282-6. http://dx.doi.org/10.1590/S1806-37132007000300009

20. Chatkin MN, Menezes AMB. Prevalência e fatores de risco para asma em uma coorte no sul do Brasil. J Pediatr, Rio de Janeiro. 2005;81(5):411-6. http://dx.doi.org/10.2223/JPED.1393

21. Luna MFG, Almeida PC, Silva MGC. Prevalência de sintomas de rinite em adolescentes de 13 e 14 anos avaliada pelo método ISAAC, na cidade de Fortaleza. Rev Bras Alerg Imunopatol. 2009;32(3):106-11.

22. Bousquet J, Dahl R, Khaltaev N. Global Alliance against Chronic Respiratory Diseases. Eur Respir J. 2007;29(2):233-9. http://dx.doi.org/10.1183/09031936.00138606

23. Pauwels RA, Buist AS, Calverley PM, Jenkins CR, Hurd SS; GOLD Scientific Committee. Global strategy for the diagnosis, management, and prevention of chronic obstructive pulmonary disease. NHLBI/WHO Global Initiative for Chronic Obstructive Lung Disease (GOLD) Workshop summary. Am J Respir Crit Care Med. 2001;163(5):1256-76. http://dx.doi.org/10.1164/ajrccm.163.5.2101039

24. Jones SC, Iverson D, Burns P, Evers U, Caputi P, Morgan S. Asthma and ageing: an end user's perspective--the perception and problems with the management of asthma in the elderly. Clin Exp Allergy. 2011;41(4):471-81. http://dx.doi.org/10.1111/j.1365-2222.2011.03718.x 